Pacific Journal of Mathematics

MPPHDENSE LINEAR TRAN NSFRRMATIO 


\section{GRAPH-DENSE LINEAR TRANSFORMATIONS}

\section{George GolightLY}

This paper extends a decomposition process of [Richard Arens, Operational calculus of linear relations, Pacific $J$. Math., 11 (1961), 9-23] for closed linear relations, $T$, on a Hilbert space to the setting in which $T$ is a linear function from a dense linear subspace of a separable normed linear complete space $S_{1}$ to an innear product space $S_{2}$. This decomposition is used in showing that such a function contracted to a suitable dense linear subspace of its initial set is the contraction of a closed linear function from a dense linear subspace of $S_{1}$ to $S_{2}$. In particular, in case the initial set of $T$ is $S_{1}$, it is shown that the contraction of $T$ to a suitable dense linear subspace of $S_{1}$ is the contraction of a continuous linear function from $S_{1}$ to $S_{2}$.

It will be supposed in the following that $\left(S_{1}, N_{1}\right)$ is a normed complex linear complete space, that $\left(S_{2}, Q_{2}\right)$ is a complex complete inner product space with $N_{2}$ the norm for $S_{2}$ corresponding to $Q_{2}$, and that $T$ is a linear function from the $N_{1}$-dense linear subspace $S$ of $S_{1}$ to $S_{2}$.

Definition. The set of all points $x$ of $S_{1}$ such that there is a sequence $z$ having $N_{1}$-limit $x$ such that $T[z]$ has $N_{2}$-limit 0 will be denoted by $Z^{\prime}(T)$ and the set of all points $x$ of $S_{2}$ such that there is a sequence $z$ having $N_{1}$-limit 0 such that $T[z]$ has $N_{2}$-limit $x$ will be denoted by $Z^{\prime \prime}(T)$. Moreover, $P^{\prime \prime}(T)$ will denote the $Q_{2}$-orthogonal projection of $S_{2}$ onto the closed linear space $Z^{\prime \prime}(T)$. The statement that $T$ is graph-dense means that $T$ is dense in the subspace $S \times$ $T(S)$ of the normed linear space $S_{1} \times S_{2}$.

Note 1. One notes that $Z^{\prime \prime}(T)$ is $\{0\}$ only in case $T$ is the contraction to $S$ of a closed linear function from a dense linear subspace of $S_{1}$ to $S_{2}$. Moreover, in case $T(S)$ lies in $Z^{\prime \prime}(T), T$ is graph-dense. Indeed, suppose $T(S)$ lies in $Z^{\prime \prime}(T)$ and $(x, y)$ is in $S \times T(S)$. Since $y-T x$ is in $Z^{\prime \prime}(T)$, there is a sequence $z$ having $N_{1}$-limit 0 such that $T[z]$ has $N_{2}$-limit $y-T x$. Hence, $x$ is the $N_{1}$-limit of $x+z$ and $y$ the $N_{2}$-limit of $T[x+z]$.

Note 2. We note the following properties of $Z^{\prime}(T)$ and $Z^{\prime \prime}(T)$.

(i) $T\left(S \cap Z^{\prime}(T)\right)$ lies in $Z^{\prime \prime}(T)$.

(ii) $T^{-1}\left(Z^{\prime \prime}(T)\right)$ lies in $Z^{\prime}(T)$.

(iii) In case $B$ is a continuous linear function from $S_{1}$ to $S_{2}$, 
$Z^{\prime \prime}(B+T)$ is $Z^{\prime \prime}(T)$.

(iv) In case $B$ is a continuous linear function from $S_{2}$ to $S_{2}$, $Z^{\prime}(T)$ lies in $Z^{\prime}(B T)$ and the closure of $B\left(Z^{\prime \prime}(T)\right)$ lies in $Z^{\prime \prime}(B T)$.

(v) In case $B$ is reversibly continuous from $S_{1}$ onto $S_{1}, Z^{\prime \prime}(T B)$ is $Z^{\prime \prime}(T)$ and $Z^{\prime}(T B)$ is $B^{-1}\left(Z^{\prime}(T)\right)$.

We shall establish (iv). Suppose $x$ is in $Z^{\prime}(T)$ and $z$ is a sequence having $N_{1}$-limit $x$ such that $T[z]$ has $N_{2}$-limit 0 . Then $B T[z]$ has $N_{2}$-limit 0 . Hence, $x$ is in $Z^{\prime}(B T)$.

Suppose $y$ is in $Z^{\prime \prime}(T)$ and $z$ is a sequence having $N_{1}$-limit 0 such that $T[z]$ has $N_{2}$-limit $y$. Then $B T[z]$ has $N_{2}$-limit $B y$; hence, $B y$ is in $Z^{\prime \prime}(B T)$. Since $Z^{\prime \prime}(B T)$ is closed and includes $B\left(Z^{\prime \prime}(T)\right)$, the closure of $B\left(Z^{\prime \prime}(T)\right)$ lies in $Z^{\prime \prime}(B T)$.

THEOREM 1. The linear function $\left(1-P^{\prime \prime}(T)\right) T$ is the contraction to $S$ of a closed linear function from a dense linear subspace of $S_{1}$ to $S_{2}$ and $P^{\prime \prime}(T) T$ is graph-dense.

Proof. Suppose $x$ is in $P^{\prime \prime}(T) T(S)$. There is a sequence $z$ having $N_{1}$-limit 0 such that $T[z]$ has $N_{2}$-limit $x$. Hence, since $P^{\prime \prime}(T) x$ is $x, P^{\prime \prime}(T) T[z]$ has $N_{2}$-limit $x$. Then, since $P^{\prime \prime}(T) T(S)$ lies in $Z^{\prime \prime}\left(P^{\prime \prime}(T) T\right), P^{\prime \prime}(T) T$ is graph-dense.

Suppose $x$ is in $Z^{\prime \prime}\left(\left(1-P^{\prime \prime}(T)\right) T\right)$. If $j$ is a positive integer, there is a point $z_{j}$ of $S$ such that $N_{1}\left(z_{j}\right)<1 / j$ and $N_{2}(x-(1-$ $\left.\left.P^{\prime \prime}(T)\right) T z_{j}\right)<1 / j$. Since $P^{\prime \prime}(T) T z_{j}$ is in $Z^{\prime \prime}(T)$, there is for each positive integer $j$ a point $w_{j}$ of $S$ such that $N_{1}\left(w_{j}\right)<1 / j$ and $N_{2}\left(P^{\prime \prime}(T) T z_{j}-T w_{j}\right)<1 / j$. Hence, $N_{2}\left(x-T\left(z_{j}-w_{j}\right)\right) \leqq N_{2}(x-(1-$ $\left.\left.P^{\prime \prime}(T)\right) T z_{j}\right)+N_{2}\left(P^{\prime \prime}(T) T z_{j}-T w_{j}\right)<2 / j$ and $N_{1}\left(z_{j}-w_{j}\right)<2 / j$. Thus, $x$ is in $Z^{\prime \prime}(T)$. Since $x$ is $\lim \left(1-P^{\prime \prime}(T)\right) T[z], P^{\prime \prime}(T) x$ is 0 . Hence, $x$ is 0 . Thus, by Note $1,\left(1-P^{\prime \prime}(T)\right) T$ is the contraction to $S$ of a closed linear function from a dense linear subspace of $S_{1}$ to $S_{2}$.

DEFINITION. In the setting that $N_{1}$ arises from an inner product $Q_{1}$ for $S_{1}$ we note that the closure in $S_{1} \times S_{2}$ of $\left(1-P^{\prime \prime}(T)\right) T$ is the common part of the closure of $T$ in $S_{1} \times S_{2}$ and the orthogonal complement in $S_{1} \times S_{2}$ of $\{0\} \times Z^{\prime \prime}(T)$, which is referred to in [2] as "the operator part" of the closure of $T$. We refer to $\left(1-P^{\prime \prime}(T)\right) T$ as the closed part, and to $P^{\prime \prime}(T) T$ as the graph-dense part, of $T$. In particular, in case $S$ is $S_{1},\left(1-P^{\prime \prime}(T)\right) T$ is referred to as the continuous part of $T$.

Note 3. In analogy with Lemma 5.2 of [1], we have that, if $T^{*}$ is the set to which $(r, s)$ belongs only in case $r$ is in $S_{2}$ and $Q_{2}(r, T \cdot)$ is continuous from $S$ to the plane and $s$ is $Q_{2}(r, T \cdot)$, i.e., $T^{*}$ is the adjoint of $T$, and $C$ is the closed part of $T$, then $T^{*}$ is 
the contraction of $C^{*}$ to the orthogonal complement of $Z^{\prime \prime}(T)$ in $S_{2}$. Indeed, if $P^{\prime \prime}(T) r$ is not 0 , there is a sequence $z$ having $N_{1}$-limit 0 such that $T[z]$ has $N_{2}$-limit $P^{\prime \prime}(T) r$; hence $\lim Q_{2}(r, T[z])$ is $N_{2}\left(P^{\prime \prime}(T) r\right)^{2}$. Hence, $r$ is not in the initial set of $T^{*}$. If $r$ is in the orthogonal complement in $S_{2}$ of $Z^{\prime \prime}(T), Q_{2}(r, T \cdot)=Q_{2}(r, C \cdot)$; hence, $(r, s)$ belongs to $T^{*}$ only in case $(r, s)$ belongs to $C^{*}$.

In particular, it should be noted that if the initial set of $T$ is $S_{1}, T^{*}$ is continuous. Suppose, now, that $L$ is a continuous linear function from $S_{2}$ to $S_{2}$. Then in order that $L T$ be continuous it is necessary and sufficient that the final set of $L^{*}$ lie in the initial set of $T^{*}$. Suppose $L T$ is continuous and $y$ is in $S_{2}$. Then the function $f$ on $S$ such that $f(x)$ is $Q_{2}\left(T x, L^{*} y\right)$ is continuous and $L^{*} y$ is in the initial set of $T^{*}$. Conversely, suppose $L^{*}\left(S_{2}\right)$ lies in the initial set of $T^{*}$. Then the initial set of $(L T)^{*}$ is $S_{2}$. Hence, $L T$ is continuous.

It may be seen that in case $C$ is a closed linear function, $D$ is a graph-dense linear function, $T$ is $C+D$, and the final set of $D$ is orthogonal in $S_{2}$ to the final set of $C$, then $Z^{\prime \prime}(D)$ includes $Z^{\prime \prime}(T)$. Indeed, if $x$ is in $Z^{\prime \prime}(T)$ and $z$ is a sequence having $N_{1}$-limit 0 such that $T[z]$ has $N_{2}$-limit $x$

$$
\left(1-P^{\prime \prime}(D)\right) x=\lim \left(\left(1-P^{\prime \prime}(D)\right) T[z]\right)=\lim C[z] .
$$

Hence, $\left(1-P^{\prime \prime}(D)\right) x$ is in $Z^{\prime \prime}(C)$. Since $C$ is closed, $Z^{\prime \prime}(C)$ is $\{0\}$. Thus, $x$ is in $Z^{\prime \prime}(D)$. In particular, in case $S$ is $S_{1}, Z^{\prime \prime}(D)$ is $Z^{\prime \prime}(T)$, $D$ is the graph-dense, and $C$ the continuous, part of $T$.

In case $S_{2}$ is the plane and $T$ is not continuous, then the kernel of $T$ is dense. We note that in this setting $Z^{\prime \prime}(T)$ is the plane, so that $T$ is graph-dense.

THEOREM 2. Suppose $S$ is $S_{1}$. Then $Z^{\prime \prime}(T)$ is finite dimensional only in case the contraction of $T$ to a dense linear subspace $W$ of $S_{1}$, having finite dimensional algebraic complement in $S_{1}$, is the contraction to $W$ of a continuous linear function from $S_{1}$ to $S_{2}$.

Proof. Suppose $Z^{\prime \prime}(T)$ is finite dimensional, $C$ is the continuous, and $D$ the graph-dense, part of $T$, and $W$ is the kernel of $D$ with closure $\bar{W}$ in $S_{1}$. Then $T$ is $C$ on $S_{1}$. Suppose $\bar{W}$ is not $S_{1}$. Suppose that $U$ is an algebraic complement in $S_{1}$ of $\bar{W}$. Then $U$ is finite dimensional. Suppose $P_{1}$ is the algebraic projection of $S_{1}$ onto $\boldsymbol{U}$ with respect to $\bar{W}$, so that $P_{1}$ is the identity on $U$ and 0 on $\bar{W}$. We note that $P_{1}$ is continuous. Indeed, with the evident interpretation of $Z^{\prime \prime}\left(P_{1}\right)$, we have $Z^{\prime \prime}\left(P_{1}\right)=Z^{\prime \prime}\left(1-P_{1}\right)$; hence, $Z^{\prime \prime}\left(P_{1}\right)$ lies in $U \cap \bar{W}$. Suppose that $P_{2}$ is the orthogonal projection of $S_{2}$ onto the orthogonal complement in $S_{2}$ of the finite dimensional subspace, 
$D(\bar{W})$. Since $D(\bar{W}) \cap D(U)$ is $\{0\}, D(\bar{W})$ is not $D\left(S_{1}\right)$. Hence, $P_{2} D$ is not 0 . Since $P_{1}$ is continuous with finite dimensional final set, $D P_{1}$ is continuous. We have the identity

$$
P_{2} D=P_{2} D P_{1}+P_{2} D\left(1-P_{1}\right)=P_{2} D P_{1} .
$$

Hence, $P_{2} D$ is continuous. We note that $Z^{\prime \prime}\left(P_{2} D\right)$ is $\{0\}, P_{2}\left(Z^{\prime \prime}(D)\right)$ lies in $Z^{\prime \prime}\left(P_{2} D\right)$, and that $D\left(S_{1}\right)$ lies in $Z^{\prime \prime}(D)$. Hence, $P_{2} D\left(S_{1}\right)$ is $\{0\}$. This is a contradiction. Thus, $\bar{W}$ is $S_{1}$.

Suppose that $W$ is a dense linear subspace of $S_{1}, W$ has finite dimensional algebraic complement in $S_{1}$, and the contraction of $T$ to $W$ is the contraction to $W$ of a continuous linear function $A$. Then $(T-A)\left(S_{1}\right)$ is finite dimensional. Since $Z^{\prime \prime}(T-A)$ lies in the closure of $(T-A)\left(S_{1}\right)$ and $(T-A)\left(S_{1}\right)$ is finite dimensional $Z^{\prime \prime}(T-A)$ is finite dimensional. Noting that $Z^{\prime \prime}(T-A)$ is $Z^{\prime \prime}(T)$, we have that $Z^{\prime \prime}(T)$ is finite dimensional. Hence, the proof is complete.

Suppose $\left(S_{1}, N_{1}\right)$ is $\left(S_{2}, N_{2}\right)$ and $S$ is $S_{1}$. In case the continuous part of $T$ is self-adjoint with respect to $Q_{2}$, then $Z^{\prime \prime}(T)$ lies in $Z^{\prime}(T)$. Indeed, $Z^{\prime}(T)$ is the kernel of the continuous part $C$ of $T$, and $Z^{\prime \prime}(T)$ lies in the orthogonal complement of $C\left(S_{1}\right)$.

THEOREM 3. Suppose $S$ is $S_{1}, C$ is the continuous part of $T$, and $D$ is the graph-dense part of $T$. Then for $x$ in $S_{1} N_{2}(T x) \geqq$ $N_{2}(C x)$. Moreover, in case $\left(S_{1}, N_{1}\right)$ is $\left(S_{2}, N_{2}\right)$ and $Z^{\prime \prime}(T)$ lies in $Z^{\prime}(T)$, for each positive integer $p N_{2}\left(T^{p} x\right) \geqq N_{2}\left(C^{p} x\right)$.

Proof. If $x$ is in $S_{1}, N_{2}(T x)^{2}=N_{2}(C x)^{2}+N_{2}(D x)^{2} \geqq N_{2}(C x)^{2}$. Suppose $\left(S_{1}, N_{1}\right)$ is $\left(S_{2}, N_{2}\right)$ and $Z^{\prime \prime}(T)$ lies in $Z^{\prime}(T)$. Then $C D$ is 0 . Hence, for each positive integer $p,(C+D)^{p}=\sum_{j=0}^{p} D^{j} C^{p-j}$. If $x$ is in $S_{1}$,

$$
\begin{gathered}
N_{2}\left(T^{p} x\right)^{2}=Q_{2}\left(\sum_{j=0}^{p} D^{j} C^{p-j} x, \sum_{j=0}^{p} D^{j} C^{p-j} x\right)=N_{2}\left(C^{p} x\right)^{2} \\
+N_{2}\left(\sum_{j=1}^{p} D^{j} C^{p-j} x\right)^{2} \geqq N_{2}\left(C^{p} x\right)^{2} .
\end{gathered}
$$

We note in the setting of Theorem 3 that in case $A$ is a continuous linear function from $S_{1}$ to $S_{2}$ which agrees with $T$ on a dense linear subspace of $S_{1}$, then for each $x$ in $S_{1} N_{2}(A x) \geqq N_{2}(C x)$.

The following lemma to Theorem 4 is proved by Kato [4, Lemma 411, p. 278].

Lemma. Suppose $(B, N)$ is a normed complex linear complete space with dual space, $\left(B^{*}, N^{*}\right)$, and that $\left(w_{p}, w_{p}^{\prime}\right)_{p=1}^{\infty}$ is a sequence in $B \times B^{*}$ such that if $p$ is a positive integer $N\left(w_{p}\right)=1, N^{*}\left(w_{p}^{\prime}\right)=$ $1, w_{p}^{\prime}\left(w_{p}\right)=1$, and for $j<p, w_{p}$ is in the kernel of $w_{j}^{\prime}$. Then if $\left(a_{p}\right)_{1}^{m}$ is a complex-sequence and $1 \leqq k \leqq m,\left|a_{k}\right| \leqq 2^{k-1} N\left(\sum_{1}^{m} a_{p} w_{p}\right)$. 
THEOREM 4. Suppose that $T$ is graph-dense and $\left(S_{1}, N_{1}\right)$ is separable. Then for each continuous linear function $C$ from $S_{1}$ to $S_{2}$ such that $C\left(S_{1}\right)$ lies in the closure of $T\left(S_{1}\right)$ and positive number $\varepsilon$, there is a dense linear subspace $S^{\prime}$ of $S_{1}$ such that for $x$ in $S^{\prime}$ $N_{2}(T x-C x) \leqq \varepsilon N_{1}(x)$.

Proof. Suppose that $\left(S_{1}, N_{1}\right)$ is separable and infinite dimensional and $e$ is an $S_{1}$-sequence, the term-set of $e$ is linearly independent, $E$ is the linear span of the term-set of $e$, and $E$ is dense in $S_{1}$. Then, with $\left(S_{1}^{*}, N_{1}^{*}\right)$ denoting the dual space of $\left(S_{1}, N_{1}\right)$, there is a sequence $\left(w_{p}, w_{p}^{\prime}\right)_{1}^{\infty}$ in $S_{1}$ satisfying the hypotheses of the lemma such that the linear span of the term-set of $\left(w_{p}\right)_{1}^{\infty}$ is $E$. Indeed, if $\left(w_{p}, w_{p}^{\prime}\right)_{1}^{n}$ is an $n$-term sequence in $S_{1} \times S_{1}^{*}$ such that if $1 \leqq j<$ $p \leqq n, N_{1}\left(w_{p}\right)=1, N_{1}^{*}\left(w_{p}^{\prime}\right)=1, w_{p}^{\prime}\left(w_{p}\right)=1, w_{j}^{\prime}\left(w_{p}\right)=0$, and the linear span of the term-set of $\left(w_{p}\right)_{1}^{n}$ is the linear span of the term-set of $\left(e_{p}\right)_{1}^{n}$, then since the common part of the set of kernels of terms of $\left(w_{p}^{\prime}\right)_{1}^{n}$ has $n$-dimensional algebraic complement in $S_{1}$, there is a point $w_{n+1}$ of norm 1 in the linear span of the term-set of $\left(e_{p}\right)_{1}^{n+1}$ and in the common part of the set of kernels of terms of $\left(w_{p}^{\prime}\right)_{1}^{n}$. Moreover, there is a point $w_{n+1}^{\prime}$ of norm 1 in $S_{1}^{*}$ such that $w_{n+1}^{\prime}\left(w_{n+1}\right)=1$. Note that $w_{n+1}$ is not in the linear span of the term-set of $\left(w_{p}\right)_{1}^{n}$. Thus, $\left(w_{p}, w_{p}^{\prime}\right)_{1}^{n+1}$ is a sequence in $S_{1} \times S_{1}^{*}$ such that if $1 \leqq j<p \leqq n+1, N_{1}\left(w_{p}\right)=1, N_{1}^{*}\left(w_{p}^{\prime}\right)=1, w_{j}^{\prime}\left(w_{p}\right)=0$, and the linear span of the term-set of $\left(w_{p}\right)_{1}^{n+1}$ is the linear span of the term-set of $\left(e_{p}\right)_{1}^{n+1}$.

Suppose that $T$ is graph-dense and $0<\varepsilon<.01$. If $j$ is a positive integer, there is a point $x_{j}$ of $S_{1}$ such that $N_{1}\left(x_{j}-w_{j}\right)<$ $(\varepsilon / 10)^{j}$ and $N_{2}\left(T x_{j}\right)<(\varepsilon / 10)^{j}$. Suppose that $A$ is the linear function on $E$ such that $A\left(\sum_{1}^{m} a_{j} w_{j}\right)=\sum_{1}^{m} a_{j} x_{j}$. Then

$$
\begin{aligned}
& N_{1}\left(A\left(\sum_{1}^{m} a_{j} w_{j}\right)-\sum_{1}^{m} a_{j} w_{j}\right) \leqq \sum_{1}^{m}\left|a_{j}\right| N_{1}\left(x_{j}-w_{j}\right) \\
& \quad \leqq \sum_{1}^{m} 2^{j-1} N_{1}\left(\sum_{1}^{m} a_{j} w_{j}\right)(\varepsilon / 10)^{j} \leqq\left(N_{1}\left(\sum_{1}^{m} a_{j} w_{j}\right) / 2\right)\left(\sum_{1}^{m}(\varepsilon / 5)^{j}\right) \\
& \quad \leqq(\varepsilon / 5) N_{1}\left(\sum_{1}^{m} a_{j} w_{j}\right) .
\end{aligned}
$$

Hence, $A$ has only one continuous linear extension $B$ to $S_{1}$ and the operator norm of $B-1$ does not exceed .01 . Hence $B$ is reversibly continuous with final set $S_{1}$. Since the linear span, $X$, of the termset of $x$ is $B(E), X$ is dense in $S_{1}$. Note that

$$
N_{2}\left(T A\left(\sum_{1}^{m} a_{j} w_{j}\right)\right)=N_{2}\left(\sum_{1}^{m} a_{j} T\left(x_{j}\right)\right) \leqq \sum_{1}^{m}\left|a_{j}\right| N_{2}\left(T x_{j}\right)
$$




$$
\begin{aligned}
& \leqq \sum_{1}^{m} 2^{j-1} N_{1}\left(\sum_{1}^{m} a_{j} w_{j}\right)(\varepsilon / 10)^{j} \leqq(1 / 2)\left(\sum_{1}^{m}(\varepsilon / 5)^{j}\right) N_{1}\left(\sum_{1}^{m} a_{j} w_{j}\right) \\
& \leqq(\varepsilon / 5) N_{1}\left(\sum_{1}^{m} a_{j} w_{j}\right) .
\end{aligned}
$$

Suppose $x$ is in $X$.

$$
N_{2}\left(T A A^{-1} x\right) \leqq(\varepsilon / 5) N_{1}\left(A^{-1} x\right) \leqq(\varepsilon / 5)(1 /(1-\varepsilon / 5)) N_{1}(x) \leqq \varepsilon N_{1}(x) .
$$

Suppose that $C$ is a continuous linear function from $S_{1}$ to $S_{2}$ such that $C\left(S_{1}\right)$ lies in the closure of $T\left(S_{1}\right)$ and $\varepsilon>0$. Then $T-C$ is graph-dense. Indeed, $Z^{\prime \prime}(T-C)$ is $Z^{\prime \prime}(T)$, the closure of $T\left(S_{1}\right)$; moreover, $(T-C)\left(S_{1}\right)$ lies in the closure of $T\left(S_{1}\right)$. There is a dense linear subspace $X$ of $S_{1}$ such that for $x$ in $X N_{2}((T-C) x) \leqq \varepsilon N_{1}(x)$.

THEOREM 5. Suppose that $\left(S_{1}, N_{1}\right)$ is separable. There is a dense linear subspace $X$ of $S_{1}$ such that the contraction of $T$ to $X$ is the contraction to $X$ of a closed linear function from a dense linear subspace of $S_{1}$ to $S_{2}$. In case $S$ is $S_{1}$, then there is a dense linear subspace $X$ of $S_{1}$ such that the contraction of $T$ to $X$ is the contraction to $X$ of a continuous linear function from $S_{1}$ to $S_{2}$.

Proof. Suppose $D$ is the graph-dense part of $T$. Then there is a dense linear subspace $X$ of $S_{1}$ such that for $x$ in $S_{1} N_{2}(D x) \leqq$ $N_{1}(x)$. Suppose that $B$ is the continuous linear extension to $S_{1}$ of the contraction of $D$ to $X$. Then $T$ is $B+(T-D)$ on $X$, the contraction to $X$ of a closed linear function. In case $S$ is $S_{1}$, then $T-D$ is the continuous part of $T$ and $T$ is continuous on $X$.

Note 4. In case $S$ is $S_{1}$ and $X$ is a dense linear subspace of $S_{1}$ such that the contraction of $T$ to $X$ is the contraction of the continuous linear function $B$ from $S_{1}$ to $S_{2}$, then the initial set of the common part of $T$ and $B$ is a dense linear subspace of $S_{1}$ lying properly in no linear subspace $Y$ of $S_{1}$ such that the contraction of $T$ to $Y$ is continuous.

Note 5. Suppose that $\left(S^{\prime}, Q^{\prime}\right)$ is a Hilbert space, $M$ is a closed linear subspace of $S^{\prime}$, and $W$ is a dense algebraic complement in $S^{\prime}$ of $M$. Then if $\phi$ is the algebraic projection of $S^{\prime}$ onto $W$ with respect to $M$ and $P$ is the orthogonal projection of $S^{\prime}$ onto $M, 1-P$ is the continuous part of $\phi$. We note that $W$ is a dense linear subspace of $S^{\prime}$ such that the contraction of $\phi$ to $W$ is continuous.

Such a dense proper linear subspace $W$ of $S^{\prime}$ having closed algebraic complement in $S^{\prime}$ is one for which there is an inner 
product $Q_{w}$ such that (W, $Q_{w}$ ) is complete and the identity function from $\left(S^{\prime}, Q^{\prime}\right)$ to $\left(W, Q_{w}\right)$ is continuous. Indeed, if $M$ is a closed algebraic complement of $W$ in $S^{\prime}$ and $P$ is the orthogonal projection of $S^{\prime}$ onto $M$, we may take $Q_{w}$ to be $Q^{\prime}((1-P) \cdot, \cdot)$. Conversely, if $W$ is a dense proper linear subspace of $S^{\prime}$ for which there is such an inner product $Q_{w}$, then the set $M$ of all limits in $S^{\prime}$ of sequences $z$ having limit 0 with respect to the norm $N_{w}$ for $W$ corresponding to $Q_{w}$ is a closed algebraic complement in $S^{\prime}$ of $W$ and, with $P$ the orthogonal projection of $S^{\prime}$ onto $M, Q_{w}$ is equivalent on $W$ to $Q^{\prime}((1-P) \cdot, \cdot)$.

Note that the formula $Q(x, y)=Q^{\prime}(x, y)+Q^{\prime}(\phi x, \phi y)$ defines an inner product $Q$ for $S^{\prime}$ such that (i) the identity function from $\left(S^{\prime}, Q\right)$ to $\left(S^{\prime}, Q^{\prime}\right)$ is continuous and (ii) $\phi$ is continuous with respect to the norm $N$ for $S^{\prime}$ corresponding to $Q$.

Of course, in this example, each power of $\phi$ is continuous on $\phi\left(S^{\prime}\right)$. More generally, it may be seen that, if $L$ is a linear function and $Q$ is an inner product for $S^{\prime}$ such that (i) the identity function from $\left(S^{\prime}, Q\right)$ to $\left(S^{\prime}, Q^{\prime}\right)$ is continuous and (ii) $L$ is continuous with respect to the norm $N$ for $S^{\prime}$ corresponding to $Q$, then there is an $N^{\prime}$-dense linear subspace $X$ of $S^{\prime}$ such that the contraction of each power of $L$ to $X$ is continuous from $\left(S^{\prime}, Q^{\prime}\right)$ to $\left(S^{\prime}, Q^{\prime}\right)$. Indeed, the condition that there be an inner product $Q$ for $S^{\prime}$ for which (i) and (ii) hold is shown in [3, Theorem 1, p. 2] to be equivalent to the existence of a positive number $b$ such that for $x$ in $S^{\prime}$ $\sum_{p=0}^{\infty} N^{\prime}\left(L^{p} x\right)^{2} / b^{p}$ converges. In this case, with $l^{2}\left(S^{\prime}\right)$ the space of all $S^{\prime}$-sequences $y$ for which $\sum_{p=0}^{\infty} N^{\prime}\left(y_{p}\right)^{2}$ converges, $\widetilde{Q}$ the inner product for $l^{2}\left(S^{\prime}\right)$ given by $\widetilde{Q}(x, y)=\sum_{p=0}^{\infty} Q^{\prime}\left(x_{p}, y_{p}\right)$, and $\widetilde{L}$ the linear function from $S^{\prime}$ to $l^{2}\left(S^{\prime}\right)$ given by $\widetilde{L}(x)_{p}=L^{p}(x) /(b)^{p / 2}$, an application of Theorem 5 to $\widetilde{L}$ yields a nonnegative number $c$ and an $N^{\prime}$-dense linear subspace $X$ of $S^{\prime}$ such that for $x$ in $X \sum_{p=0}^{\infty} N^{\prime}\left(L^{p} x\right)^{2} / b^{p}=$ $\widetilde{Q}(\widetilde{L} x, \widetilde{L} x) \leqq c \widetilde{N}^{\prime}(x)^{2}$.

\section{REFERENCES}

1. Richard Arens, Operational calculus of linear relations, Pacific J. Math., 11 (1961), 9-23.

2. Earl A. Coddington, Selfadjoint subspace extensions of nondensely defined symmetric operators, Bull. Amer. Math. Soc., 79 (1973), 712-715.

3. George Golightly, Shadow and inverse-shadow inner products for a class of linear transformations, pre-print, Auburn Univ., (1978).

4. Tosio, Kato Perturbation theory for nullity, deficiency and other quantities of linear operators, J. d' Analyse Math., 6 (1958), 273-322.

Received May 30, 1978. 



\section{PACIFIC JOURNAL OF MATHEMATICS}

\section{EDITORS}

Donald BABBITT (Managing Editor)

University of California

Los Angeles, CA 90024

HUGo RossI

University of Utah

Salt Lake City, UT 84112

C. C. MOORE and ANDREW OGG

University of California

Berkeley, CA 94720

\section{J. DuGUNDJI}

Department of Mathematics

University of Southern California

Los Angeles, CA 90007

R. FINN and J. Milgram

Stanford University

Stanford, CA 94305

ASSOCIATE EDITORS
E. F. BECKENBACH
B. H. NeumanN
F. WOLF
K. YoSHIDA

\section{SUPPORTING INSTITUTIONS}

\author{
UNIVERSITY OF BRITISH COLUMBIA \\ CALIFORNIA INSTITUTE OF TECHNOLOGY \\ UNIVERSITY OF CALIFORNIA \\ MONTANA STATE UNIVERSITY \\ UNIVERSITY OF NEVADA, RENO \\ NEW MEXICO STATE UNIVERSITY \\ OREGON STATE UNIVERSITY \\ UNIVERSITY OF OREGON
}

\author{
UNIVERSITY OF SOUTHERN CALIFORNIA \\ STANFORD UNIVERSITY \\ UNIVERSITY OF HAWAII \\ UNIVERSITY OF TOKYO \\ UNIVERSITY OF UTAH \\ WASHINGTON STATE UNIVERSITY \\ UNIVERSITY OF WASHINGTON
}

The Supporting Institutions listed above contribute to the cost of publication of this Journal, but they are not owners or publishers and have no responsibility for its content or policies.

Mathematical papers intended for publication in the Pacific Journal of Mathematics should be in typed form or offset-reproduced, (not dittoed), double spaced with large margins. Please do not use built up fractions in the text of the manuscript. However, you may use them in the displayed equations. Underline Greek letters in red, German in green, and script in blue. The first paragraph or two must be capable of being used separately as a synopsis of the entire paper. Please propose a heading for the odd numbered pages of less than 35 characters. Manuscripts, in triplicate, may be sent to any one of the editors. Please classify according to the scheme of Math. Reviews, Index to Vol. 39. Supply name and address of author to whom proofs should be sent. All other communications should be addressed to the managing editor, or Elaine Barth, University of California, Los Angeles, California, 90024.

50 reprints to each author are provided free for each article, only if page charges have been substantially paid. Additional copies may be obtained at cost in multiples of 50 .

The Pacific Journal of Mathematics is issued monthly as of January 1966. Regular subscription rate: $\$ 84.00$ a year (6 Vols., 12 issues). Special rate: $\$ 42.00$ a year to individual members of supporting institutions.

Subscriptions, orders for numbers issued in the last three calendar years, and changes of address should be sent to Pacific Journal of Mathematics, P.O. Box 969, Carmel Valley, CA 93924, U.S.A. Older back numbers obtainable from Kraus Periodicals Co., Route 100, Millwood, NY 10546.

PUBLISHED BY PACIFIC JOURNAL OF MATHEMATICS, A NON-PROFIT CORPORATION

Printed at Kokusai Bunken Insatsusha (International Academic Printing Co., Ltd.). 8-8, 3-chome, Takadanobaba, Shinjuku-ku, Tokyo 160, Japan.

Copyright (C) 1979 by Pacific Journal of Mathematics Manufactured and first issued in Japan 


\section{Pacific Journal of Mathematics}

\section{Vol. 82 , No. 2 \\ February, 1979}

Krishnaswami Alladi and Paul Erdős, On the asymptotic behavior of large prime

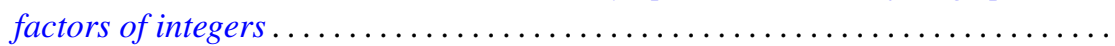

Alfred David Andrew, A remark on generalized Haar systems in $L_{p}$,

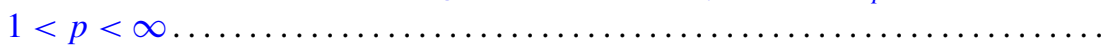

John M. Baker, A note on compact operators which attain their norm . . ........

Jonathan Borwein, Weak local supportability and applications to

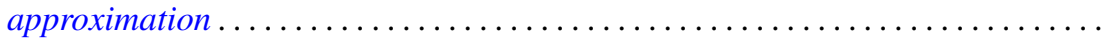

Tae Ho Choe and Young Soo Park, Wallman's type order compactification ........

Susanne Dierolf and Ulrich Schwanengel, Examples of locally compact

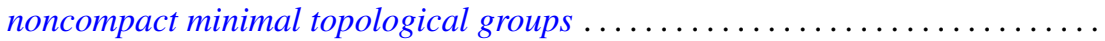

Michael Freedman, A converse to (Milnor-Kervaire theorem) $\times R$ etc. . . . . . . .

George Golightly, Graph-dense linear transformations ..................

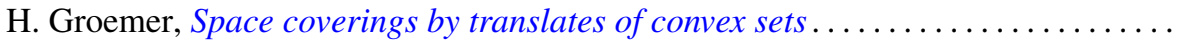

Rolf Wim Henrichs, Weak Frobenius reciprocity and compactness conditions in

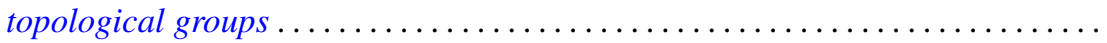

Horst Herrlich and George Edison Strecker, Semi-universal maps and universal

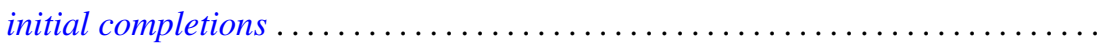

Sigmund Nyrop Hudson, On the topology and geometry of arcwise connected,

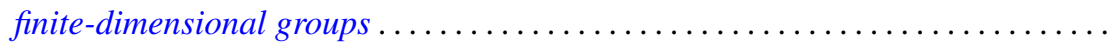

K. John and Václav E. Zizler, On extension of rotund norms. II .............

Russell Allan Johnson, Existence of a strong lifting commuting group of transformations. II.

Bjarni Jónsson and Ivan Rival, Lattice varieties covering the smallest nonmodular variety

Grigori Abramovich Kolesnik, On the order of Dirichlet L-functions .

Robert Allen Liebler and Jay Edward Yellen, In search of nonsolvable groups of

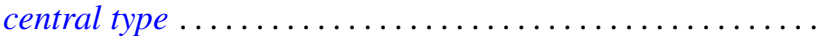

Wilfrido Martínez T. and Adalberto Garcia-Maynez Cervantes, Unicoherent plane Peano sets are $\sigma$-unicoherent ...

M. A. McKiernan, General Pexider equations. I. Existence of injective

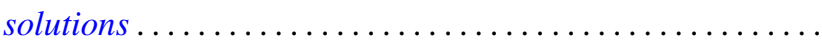

M. A. McKiernan, General Pexider equations. II. An application of the theory of webs.

Jan K. Pachl, Measures as functionals on uniformly continuous functions . .

Lee Albert Rubel, Convolution cut-down in some radical convolution algebras ...

Peter John Slater and William Yslas Vélez, Permutations of the positive integers

with restrictions on the sequence of differences. II . . .

Raymond Earl Smithson, A common fixed point theorem for nested spaces ....

Indulata Sukla, Generalization of a theorem of McFadden .... . . .

Jun-ichi Tanaka, A certain class of total variation measures of analytic measures.

Kalathoor Varadarajan, Modules with supplements .............. 